\title{
Honey bees communicate distance via non-linear waggle duration functions
}

\author{
Patrick Laurenz Kohl ${ }^{\text {Corresp., } 1}$, Benjamin Rutschmann ${ }^{1}$ \\ ${ }^{1}$ Department of Animal Ecology and Tropical Biology, Biocenter, University of Würzburg, Würzburg, Germany \\ Corresponding Author: Patrick Laurenz Kohl \\ Email address: patrick.kohl@uni-wuerzburg.de
}

Honey bees (genus Apis) can communicate the approximate location of a resource to their nestmates via the waggle dance. The distance to a goal is encoded by the duration of the waggle phase of the dance, but the precise shape of this distance-duration relationship is ambiguous: earlier studies (before the 1990s) proposed that it is non-linear, with the increase in waggle duration flattening with distance, while more recent studies suggested that it follows a simple linear function (i. e. a straight line). Strikingly, authors of earlier studies trained bees to much longer distances than authors of more recent studies, but unfortunately they usually measured the duration of dance circuits (waggle phase plus return phase of the dance), which is only a correlate of the bees' distance signal. We trained honey bees ( $A$. mellifera carnica) to visit sugar feeders over a relatively long array of distances between 0.1 and $1.7 \mathrm{~km}$ from the hive and measured the duration of both the waggle phase and the return phase of their dances from video recordings. The distancerelated increase in waggle duration was better described by a non-linear model with a decreasing slope than by a simple linear model. The relationship was equally well captured by a model with two linear segments separated at a "break-point" at $1 \mathrm{~km}$ distance. In turn, the relationship between return phase duration and distance was sufficiently well described by a simple linear model. The data suggest that honey bees process flight distance differently before and beyond a certain threshold distance. While the physiological and evolutionary causes of this behavior remain to be explored, our results can be applied to improve the estimation of honey bee foraging distances based on the decoding of waggle dances. 


\section{Honey bees communicate distance via non-linear}

2 waggle duration functions

3

4

5

6

7

8

9

10

11

12

13

14

15

16

17

18

19

20

21

22

23

24

25

26

27

28

29

30

31

32

33

34

35

36

37

38

39

Patrick L. Kohl and Benjamin Rutschmann

Department of Animal Ecology and Tropical Biology, Biocenter, University of Würzburg, Würzburg, Germany

Corresponding Author:

Patrick L. Kohl

Am Hubland, 97074 Würzburg, Germany

Email address: patrick.kohl@uni-wuerzburg.de

\section{Abstract}

Honey bees (genus Apis) can communicate the approximate location of a resource to their nestmates via the waggle dance. The distance to a goal is encoded by the duration of the waggle phase of the dance, but the precise shape of this distance-duration relationship is ambiguous: earlier studies (before the 1990s) proposed that it is non-linear, with the increase in waggle duration flattening with distance, while more recent studies suggested that it follows a simple linear function (i. e. a straight line). Strikingly, authors of earlier studies trained bees to much longer distances than authors of more recent studies, but unfortunately, they usually measured the duration of dance circuits (waggle phase plus return phase of the dance), which is only a correlate of the bees' distance signal. We trained honey bees (A. mellifera carnica) to visit sugar feeders over a relatively long array of distances between 0.1 and $1.7 \mathrm{~km}$ from the hive and measured the duration of both the waggle phase and the return phase of their dances from video recordings. The distance-related increase in waggle duration was better described by a non-linear model with a decreasing slope than by a simple linear model. The relationship was equally well captured by a model with two linear segments separated at a "break-point" at $1 \mathrm{~km}$ distance. In turn, the relationship between return phase duration and distance was sufficiently well described by a simple linear model. The data suggest that honey bees process flight distance differently before and beyond a certain threshold distance. While the physiological and evolutionary causes of this behavior remain to be explored, our results can be applied to improve the estimation of honey bee foraging distances based on the decoding of waggle dances.

\section{Introduction}

Honey bees can use the waggle dance to communicate the approximate locations of resources to their nestmates (von Frisch, 1965; Dyer, 2002). Upon returning from a successful trip a worker honey bee may perform sequences of dance circuits on the comb alternating to the left and to the

Peer] reviewing PDF | (2020:12:56277:1:1:NEW 7 Mar 2021) 
40 right. Each circuit is composed of the waggle phase, in which the bee walks straight and shakes

41 its abdomen, and the return phase, in which it turns back and walks to the starting point. It is the

42 waggle phase of the dance which conveys the information about the direction and the distance of

43 the resource in the field (Michelsen et al., 1992; Dyer, 2002). A foraging honey bee uses the

44 sun's position or the polarization patterns of sunlight on the blue sky to obtain compass

45 information (von Frisch, 1965). In the dance, the direction to the resource in relation to the nest

46 is encoded by the bee's body orientation during the waggle runs. When dancing on a horizontal

47 surface in the open, a bee refers to celestial cues and directly "points" its dances towards the

48 goal. When dancing on the vertical comb inside the dark hive, a dancer refers to gravity, and its

49 body orientation with reference to the upwards direction encodes the direction to the resource

50 with reference the sun's current position over the horizon. Provided with information of the

51 context of a dance, a human observer can unambiguously infer the compass direction indicated

52 by a dancer of any honey bee species (Lindauer, 1956; see Dyer, 1991, 2002 or Preece \&

53 Beekman, 2014 for reviews of direction encoding in the waggle dance). The interpretation of the

54 bees' distance signal is less straightforward. Foragers visually gauge the length of the flight path

55 to the goal based on the amount of image motion perceived during flight (optic flow) and encode

56 it in the duration of the waggle phase, with longer waggle phases indicating further distances

57 (Esch and Burns, 1996; Srinivasan, 2000; Esch et al., 2001; De Marco and Menzel, 2005).

58 However, there is no universal distance-code and different (sub)species of honey bee can vary

59 substantially in the slopes of their distance-duration functions (Lindauer, 1956; Bosch, 1957;

60 Kohl et al., 2020). Apart from that, it is not even clear what is the precise shape of the distance-

61 duration relationship. The current view is that waggle duration increases with foraging distance

62 in a simple linear fashion (Schürch et al., 2013, 2016, 2019; Kohl et al., 2020). Interestingly,

63 Karl von Frisch, the discoverer of the spatial information content of the waggle dance, and his

64 colleagues had originally proposed a different shape of the function (v. Frisch and Jander, 1957;

65 von Frisch and Kratky, 1962; von Frisch, 1965). Based on several early experiments conducted

66 between 1945 and 1960 it was established that the distance communication function is non-linear

67 in that the slope of waggle duration flattens beyond a certain distance (figure 1A) (von Frisch,

68 1965). Such a shape was independently corroborated later by a study of Visscher (Visscher,

69 1982).

70 To identify possible reasons for this apparent contradiction between the original and the current

71 view, we closely inspected all studies available to us in which Western honey bees (Apis

72 mellifera) of European origin were trained to feeders to infer the distance function of the waggle

73 dance (table 1). This revealed that authors of earlier studies (performed before the 1990s)

74 generally trained the bees to much further distances (usually well beyond $1 \mathrm{~km}$ ) than authors of

75 more recent studies. In fact, there seems to be an association between the maximum distances the

76 bees were trained, and whether curvature was found in the distance communication function

77 (figure 1B). Unfortunately, this relationship is confounded by another variable differing between

78 earlier and more recent studies: authors of earlier studies usually considered a correlate of the

79 distance signal, the duration of whole dance circuits (waggle phase plus return phase), instead of 
80

81

82

83

84

85

86

87

88

89

90

91

92

93

94

95

96

97

98

99

100

101

102

103

104

105

106

107

108

109

110

111

112

113

114

115

116

117

118

119

measuring waggle phase duration. This was done mainly because circuit duration can be directly measured with a stopwatch, whereas one needs to record dances on video to precisely measure waggle phase durations, which was expensive before digital video cameras became readily available. Consequently, there are two explanations for the apparent ambiguity concerning the shape of the distance communication function. Perhaps, curvature indeed exists in the distancewaggle duration function, but it can only be clearly detected when the bees are trained significantly further than $1 \mathrm{~km}$ from the hive. Alternatively, early studies found curvature in the distance-circuit duration relationship only because there is curvature in the relationship between distance and the return phase duration, not the waggle phase duration.

There are two historical studies which could have solved the problem since bees were trained to feeders at relatively long distances, and the three durations components of the resulting dances were determined (v. Frisch and Jander, 1957; Wenner, 1962). Unfortunately, these studies seem to have conflicting results. Von Frisch and Jander (v. Frisch and Jander, 1957) trained bees to feeders at seven distances between 200 and 4500 meters from the hive and made time measurements from film recordings. Their data suggested that there is curvature in the distancewaggle duration function and little or no curvature in the distance-return duration function (figure 2). Based on this study, von Frisch concluded that the shape of the circuit duration function well reflects the shape of the waggle duration function (von Frisch, 1965). Wenner (Wenner, 1962) trained bees to feeders at 16 distances between 31 and $1230 \mathrm{~m}$ from the hive and determined the different duration components from sound recordings. He found that the waggle phase duration followed essentially a straight line, while there was clear curvature in the relationship between distance and return phase duration (figure 2). However, it is unclear whether Wenner's results actually contradict the data of von Frisch and Jander. On the one hand, Wenner's maximum training distance of $1230 \mathrm{~m}$ was perhaps too short to detect the curvature in the waggle duration function. On the other hand, Wenner reports that sugar concentration was gradually rising during his experiment because of hot temperatures. Since honey bees perform quicker return phases when dancing for food sources that are higher in quality (Seeley, Mikheyev and Pagano, 2000; Hrncir et al., 2011; Łopuch and Tofilski, 2020), the gradual increase in sugar concentration could explain why the bees reduced the return phase duration at long feeder distances (that were reached later in the experiment).

To solve the ambiguity about the distance-duration relationship of the waggle dance, we performed a new experiment. We trained bees to forage at an array of feeder positions up to a relatively long distance of $1.7 \mathrm{~km}$ from the hive and determined both the waggle phase duration and the return phase duration of their corresponding dances from video recordings. Then, we fit three types of models to the distance-duration relationships - a linear model, a non-linear model, and a break-point model - and compared their goodness-of-fit to determine which captured the distance-duration relationships best.

\section{Materials \& Methods}

\section{Feeder training experiment}


120 We conducted the experiment in the Steigerwald, a forested hill region located between the cities 121 of Würzburg and Nuremberg in Germany. Permits to work in the publicly-owned forest were granted by the Bavarian State forest administration in Ebrach.

125

126

127

128

129

130

131

132

133

134

135

136

137

138

139

140

141

142

143

144

145

146

147

148

149

150

151

152

153

154

155

156

157

158

159

Since honey bees use optic flow to estimate the distance flown to a goal, variation in the visual appearance of the terrain can affect their distance estimations (Tautz et al., 2004). Although the visual odometer is thought to be quite robust with respect to the contrast variation the bees typically encounter when foraging (Si, Srinivasan \& Zhang, 2003; George et al., 2020), we wanted to make sure that the effect of foraging distance on dance behavior was not confounded by variation in the terrain. The forest location allowed us to train the bees in a homogenous terrain along a straight gravel road through beech forest. With this setting, the bees encountered the same scenery of mature forest at every section of their flight paths, so that variation in waggle duration between feeder locations was attributable to changes in feeder distance alone. We worked from 8-12 August 2019, when natural forage was scarce so that the bees could easily be lured to an artificial food source. Two days before feeder training, we transferred a colony of A. mellifera carnica housed in an observation hive to the experimental site (N 49.8618, E 10.5366). The colony was queen right and had brood in all stages. The hive, which we mounted onto a wooden stand, contained two frames stacked over each other (standard Zander frames) and had glass windows on both sides which could be covered by wooden boards. A wedge placed behind the hive entrance forced homecoming bees to enter the colony on one side of the combs. This made it possible to capture most dances while only recording one side of the colony. During video observations, we used a light-proof cloth spread out over the hive to prevent direct sunlight from entering the colony.

To train the bees to feeders at varying distances from the hive we first offered the bees a piece of wax comb filled with concentrated sucrose solution in front of the hive entrance. Once the bees discovered the bait, we transferred the feeder with the bees to a small colored table at kneeheight and moved this feeding station step by step until we reached the first test location at 100 $\mathrm{m}$ distance (determined with a hand-held GPS device). We let the bees forage for around 30 minutes to adjust to the location before we started dance observations. During that time, we labelled each individual bee visiting the feeder with a unique combination of shellac color paints on the thorax and/or abdomen. Then we video recorded their dances back at the hive at a frame rate of $50 \mathrm{~Hz}$ using a digital camcorder (Panasonic HC-X929). The sequence of moving the feeder, marking bees and video recording was repeated for eight other feeder locations, each 200 $\mathrm{m}$ further from the hive up to the final feeder distance of $1.7 \mathrm{~km}$. Throughout the experimental period weather conditions were suitable for honey bee foraging (no rain, temperatures between 18 and $28^{\circ} \mathrm{C}$, mean wind speed of only $2.7 \mathrm{~m} / \mathrm{s}$ outside the forest; data from the nearest weather station: Agrarmetereologie Bayern, weather station Kleingressingen, www.wetter-by.de). At all feeder distances we offered the same sucrose solution ( $2 \mathrm{M}$, scented with star anise extract) so that the relative attractiveness of our artificial food source was only affected by the distance to the hive and by potential fluctuations in the colony's parallel intake of other, natural nectar

Peer) reviewing PDF | (2020:12:56277:1:1:NEW 7 Mar 2021) 
160

161

162

163

164

165

166

167

168

169

170

171

172

173

174

175

176

177

178

179

180

181

182

183

184

185

186

187

188

189

190

191

192

193

194

195

196

197

198

sources. However, we believe that the natural resource environment was largely stable since the bees did not perform many waggle dances for other food sources during the experiment. At the feeder, the bees remained undisturbed by predators or competitors (e.g. wasps or ants) and there was no indication that bees from another colony visited our feeder (i.e. the foragers marked at the feeder showed up at the hive and we did not observe any fights between bees).

\section{Dance measurements}

We first screened the videos and listed all dances of all individually labelled bees that had been recorded. There were 90 dances of a total of 56 identifiable bee individuals captured on the videos. For the analyses, we selected dances in such a way that we represented every bee once while at the same time balancing the number of dances per feeder distance. This resulted in a sample of 56 dances (5-8 dances per distance), each performed by a different bee. Considering that different workers from a single colony vary in their distance communication (von Frisch, 1965; Schürch et al., 2016), this sampling scheme guaranteed that the distance communication function we inferred was not biased towards any individual bee with extreme behavior. Following the basic protocol of Couvillon et al. (Couvillon et al., 2012) we timed the duration of four consecutive waggle phases per dance, defining waggle duration as the time lapse between the first video frame in which a bee had started shaking its abdomen (identified as the moment when the image of the bee got blurred) until the first frame in which it had stopped waggling again. We also determined the duration of four subsequent return phases (the time lapses between two waggle runs). The measurements were performed manually on a computer using the program utilius fairplay 5 (ccc software, Leipzig, Germany). We averaged to get the mean waggle phase and the mean return phase duration per dance, respectively. By adding mean waggle phase duration and mean return phase duration we obtained the dances' mean circuit duration. These data, one value of waggle phase, return phase and circuit duration per dance, were considered for the analyses.

\section{Comparisons of distance-duration models}

For each of the three time-variables of the dances - waggle phase duration $\left(t_{w}\right)$, return phase duration $\left(t_{r}\right)$, and their sum, circuit duration $\left(t_{c}\right)$ - we tested whether the relationships with feeder distance $(d)$ were sufficiently described by simple linear regression models, or whether more complex models (allowing for changes in slope over the range of distances) resulted in better fits. All analyses were performed in R version 3.6.2 (R Core Team, 2019). Simple linear regressions, which we refer to as "linear models", were fit using the "lm" function of the stats package. As an alternative model, which allowed for a gradually decreasing slope of duration versus distance and which we refer to as the "non-linear model", we fit a formula proposed by Frisch and Kratky (von Frisch and Kratky, 1962) using the "nls" function from the "stats" package:

$$
t=a+\frac{b}{c}\left(1-e^{-c d}\right)
$$

Peer] reviewing PDF | (2020:12:56277:1:1:NEW 7 Mar 2021) 
199

200

201

202

203

204

205

206

207

208

209

210

211

212

213

214

215

216

217

218

219

220

221

222

223

224

225

226

227

228

229

230

231

232

233

234

235

236

237

238

Frisch and Kratky deduced this formula based on behavioral and physiological considerations, and it well reflected the distance-duration relationships obtained experimentally by Frisch and Jander (v. Frisch and Jander, 1957). As another alternative to the linear model, we fit a segmented linear regression with two linear equations and a "break-point" using the function "segmented" (from the eponymous R-package; Vito and Muggeo, 2008), which we refer to as the "segmented model".

We evaluated and compared the models based on three criteria. First, we checked whether the predictions showed any biases across the range of feeder distances, i.e. whether the models systematically under- or overestimated the duration components at any point (aided by the inspection of plots of fitted values versus residuals); second, we computed the Pearson's correlation coefficient $r$ for correlations between predicted values and original observations, allowing us to compare how well the models fit the data; and third, we compared the models' Akaike information criterion values (for small sample sizes, AICctab-function of the bbmle package (Bolker et al. 2017), allowing us to check which of the three models would optimize fit versus complexity.

\section{Results}

Waggle phase durations increased by 5.4 times from $0.41 \pm 0.10$ seconds at $100 \mathrm{~m}$ to $2.20 \pm 0.19$ seconds at $1.7 \mathrm{~km}$ (throughout this section, results are reported as means $\pm \mathrm{SDs}$ ). The relationship clearly deviated from simple linearity, as the slope flattened with increasing distance from the hive (Fig. 3A). Accordingly, our non-linear model, which allowed for a continuous decrease in slope, had a better fit than the linear model ( $r=0.946$ versus $r=0.935, \Delta$ AICc: 7.7$)$. The segmented model, which divided the relationship into two linear regressions separated at a break-point, fit the data similarly well as the non-linear model ( $r=0.947, \Delta \mathrm{AICc}: 1.5)$. The breaking point was estimated to lay at around $1 \mathrm{~km}$ distance (table 2).

Return phase durations also increased with distance but at a slower rate and with more variability than waggle phase durations. At feeder distances between $100 \mathrm{~m}$ and $1.7 \mathrm{~km}$, return phase durations rose from $1.40 \pm 0.33$ seconds to $2.31 \pm 0.29$ seconds (a 1.7-fold increase, Fig 3B). The relationship was sufficiently described by a simple linear model (table 2 ).

Being the combination of the waggle phase and the return phase, circuit duration increased by 2.5 times from $1.81 \pm 0.27$ seconds to $4.51 \pm 0.37$ seconds between $100 \mathrm{~m}$ and $1.7 \mathrm{~km}$. Given the curvature in the function relating waggle phase duration to distance, the distance related increase in circuit duration was also better described by the non-linear model than by the linear model (table 2). Again, the segmented model resulted in a fit comparable to that of the non-linear model. For circuit durations, the break-point was estimated to lay at $0.7 \mathrm{~km}$ distance.

\section{Discussion}

Honey bees communicated via a non-linear distance-waggle duration function

By training honey bees to feeders up to a distance of $1.7 \mathrm{~km}$ from the hive and by timing their corresponding recruitment dances, we found that their distance function is non-linear in the sense 
239 that the increase in waggle duration flattens beyond a certain distance from the hive. While this

240 confirms the original concept of von Frisch (von Frisch, 1965), our results are in seeming

241 contrast to more recent studies, who proposed a simple linear relationship (e.g. Schürch et al.,

242 2013, 2019). In the early studies of the waggle dance, researchers trained bees to much further

243 distances (well beyond $1 \mathrm{~km}$ ) than was done in more recent studies. However, they usually only

244 determined the relationship between distance and the duration of whole dance circuits (waggle

245 phase plus return phase), taking it as a proxy for the bees' distance communication function. This

246 created two problems. On the one hand, it could be that the distance function does not show

247 curvature before a certain threshold distance, so that the non-linearity can simply not be detected

248 when the bees are only trained to feeders at short distances from the hive. On the other hand it

249 was unclear whether the non-linear shape of the circuit duration function was actually due to

250 non-linearity of the distance function of waggle duration or whether it stemmed from potential

251 curvature in the relationship between distance and the return phase of the dance. Our study

252 solved this ambiguity as we trained bees significantly further than has been done in any of the

253 other recent studies, and as we timed both the waggle phase and the return phase of the dance.

254 This clearly revealed that the distance-waggle duration relationship is non-linear when

255 considered over a large range of distances. However, since the slope of waggle duration only

256 flattens significantly after about $1 \mathrm{~km}$ distance from the hive, we also confirmed that the

257 distance-waggle duration relationship is well captured by a simple linear model for a range of

258 closer distances.

259

260 Interestingly, the relationship between return phase duration and distance was sufficiently

261 described by a simple linear model. This supports the original assumption of $v$ Frisch that the

262 curvature detected in the distance-circuit duration curves is attributable to non-linearity in the

263 waggle duration signals (v. Frisch and Jander, 1957; von Frisch, 1965). Honey bees tune the

264 duration of the return phase in response to resource quality so that they have quicker return

265 phases when a resource is more profitable (Seeley, Mikheyev and Pagano, 2000; Hrncir et al.,

266 2011; Łopuch and Tofilski, 2020). Therefore, a possible explanation for why mean return phases

267 increased with distance in our experiment is that the bees' ratings of the feeder's profitability

268 lowered while we moved it away from the hive. Since we kept the sugar concentration constant

269 at $2 \mathrm{M}$, such a reduction in profitability rating would have been due to the longer distances the

270 bees needed to travel to collect the same resource, or due to a gradual saturation of the colony

271 during the experiment. Alternatively, the increasing return phase duration can simply be

272 explained by the distance-related increase in waggle duration. After a waggle run, the bees walk

273 back to their approximate starting point to keep their position on the comb while dancing. Given

274 that the bees walk further stretches on the comb when waggle durations are longer (Heran,

275 1956), they also need to walk back further during the return phase resulting in longer return

276 durations.

277 Our distance-waggle duration function is similar in shape to a function fit through the historical

278 reference data by Frisch and Jander (v. Frisch and Jander, 1957), who determined waggle 
279 durations at seven feeder distances between 0.2 and $4.5 \mathrm{~km}$ (Fig. 4A, the red line and black line). 280 Furthermore, our data on the distance-circuit duration relationship are in good accordance with 281 the data presented by v. Frisch (von Frisch, 1965) (which are averages based on several studies) 282 (Figure 4B). Especially there is a smooth transition between the values at our furthest training 283 distances and the data presented by v. Frisch from $2 \mathrm{~km}$ onwards. The general similarity was 284 expected given that the bees used by us and those used by v. Frisch and his colleagues belonged 285 to same subspecies of bees.

286

287

288

289

290

291

292

293

294

295

296

297

298

299

300

301

302

303

304

305

306

307

308

309

310

311

312

313

314

315

316

317

318

\section{The general shape of the distance communication function of honey bees}

The insight the distance-waggle duration function is non-linear and that the form of the circuit duration curve represents the form of the waggle duration curves allows us to consider the results of both old and new studies to outline the general shape of the distance communication function. In referring to it as the "general shape", we mean that it probably applies to the distance functions of all honey bee species, although there are population- and species-specific differences in the slopes of these functions (Kohl et al., 2020). The evidence for this stems from experiments with four non-European honey bee (sub)species: in studying dances of

A. mellifera scutellata from tropical Africa, Schneider (Schneider, 1989) found that in this honey bee subspecies the relationship between distance and waggle duration was better described by a curve with a decreasing slope than by a straight line; and in training foragers of the three Asian honey bee species A. florea, A. cerana and A. dorsata to feeders at relatively long distances in Thailand, Dyer and Seeley (Dyer and Seeley, 1991) found that the distance-circuit duration curves of each of the three species followed a segmented function with the first linear segment being steeper than the second one.

Von Frisch and Kratky (von Frisch and Kratky, 1962) proposed that the distance-waggle duration function has the shape of a curve with a continuously flattening slope. From a physiological perspective there would be an obvious proximate explanation for such a model. When flying to a foraging site, a honey bee perceives optic flow which she uses to estimate flight distance (Esch and Burns, 1996; Srinivasan, 2000; Esch et al., 2001; De Marco and Menzel, 2005). With increasing flight distance, however, the neurons processing the sustained optic flow stimulus might decrease their responsiveness (Maddess and Laughlin, 1985; Clifford and Ibbotson, 2002; Taylor and Krapp, 2007). As a consequence, segments of the flight path that are travelled later during a trip would lead to lower distance estimates than earlier segments. This form of neural fatigue would result into the proposed continuously flattening curve (von Frisch and Kratky, 1962). The curve would then be interpretable as the result of a physiological constraint or insufficiency.

However, the available data rather suggest a different scenario, namely that the distance-waggle duration function has two linear phases - a steeper phase for closer distances and a lower phase for longer distances - mediated by a rather sudden, curved transition around a threshold distance. There are several indications that such a segmented relationship captures the process better than a continuously flattening slope. First, all studies in which bees were trained to relatively short 
319

320

321

322

323

324

325

326

327

328

329

330

331

332

333

334

335

336

337

338

339

340

341

342

343

344

345

346

347

348

349

350

351

352

353

354

355

356

357

distances (up to around $1 \mathrm{~km}$ distance) found that the relationship was well described by a simple linear model (Sasaki, Takahashi and Sato, 1993; Tautz et al., 2004; Su et al., 2008; Schürch et al., 2013, 2016, 2019; Kohl et al., 2020). Second, the data from studies in which bees were trained well below $1 \mathrm{~km}$ suggest that waggle duration (or circuit duration) increases linearly over the range of far distances from about $1 \mathrm{~km}$ onwards (v. Frisch and Jander, 1957; von Frisch, 1965) (figure $4 \mathrm{~A}$ and B). Third, the average data on the distance-circuit duration relationship which are based on several studies compiled by v. Frisch suggest a sudden break-point rather than a continuously flattening slope (von Frisch, 1965)(figure 1A, figure 4B). And fourth, our data were fitted equally well by a segmented model as with a non-linear continuous curve (table 2). Of course, it is hard to believe that a behavioral process shows a clear-cut break-point. Even if individual bees possessed distance-curves with such a sudden break-point, variation between individuals of a colony and variation between colonies of a populations would lead to a function with a "smooth" turning point on the colony or population level, respectively. The segmented function seems to be a good model, though. It suggests that the bees process distance differently before and beyond a certain threshold distance. While the physiological basis for this might well involve a sensory adaptation process, a segmented relationship could not simply be explained by a mere physiological constraint. Instead, this shape suggests that it has some adaptive value.

Dancing honey bees have the problem that they are limited in how long waggle phases can potentially be since very long waggle runs become difficult to follow by potential recruits and take a lot of time during which the dancers cannot collect food themselves. Consequently, they face a trade-off between the steepness of their distance-waggle duration functions (which affects the communication precision) and the maximum foraging distances which they can indicate in their dances (Dyer, 2002; Kohl et al., 2020). In line with this, honey bee (sub)species with smaller foraging ranges evolved steeper distance-duration functions, at least when the first segment of their distance function is considered (Kohl et al., 2020). However, the segmented distance function could reduce some pressure from this trade-off between communication precision and communication range: with a segmented distance function, the bees would have a first steeper segment to communicate more precisely over a range of closer distances from their hive, while the second lower segment would still allow them to communicate foraging sites at far distances, if necessary. As an extension of the adaptive-tuning hypothesis for the evolution of distance dialects (Dyer and Seeley, 1991; Kohl et al., 2020), the prediction is that honey bee populations with longer foraging ranges have distance functions with break-points at further distances. The available data are in line with this prediction: temperate honey bees, which have larger foraging ranges than tropical honey bees (Kohl et al., 2020), also have break-points at further distances $(\sim 1 \mathrm{~km})$ than tropically adapted honey bees (break-points between 200 and 560 m (Schneider, 1989; Dyer and Seeley, 1991).

\section{Caveats}

Peer] reviewing PDF | (2020:12:56277:1:1:NEW 7 Mar 2021) 
358

359

360

361

362

363

364

365

366

367

368

369

370

371

372

373

374

375

376

377

378

379

380

381

382

383

384

385

386

387

388

389

390

391

392

393

394

395

396

397

Before reasonable analyses of the proximate and ultimate causes can be performed, the apparently non-linear distance communication functions need to be explored and described in more detail. For example, even though we trained bees further away from the hive than it was done in any other study since the experiment of Lindauer in 1960 (see table 1), our maximum training distance of $1.7 \mathrm{~km}$ was clearly not long enough to unambiguously determine the shape of the distance function at long distances because temperate honey bees are known to forage at distances more than $10 \mathrm{~km}$ away from the hive (Beekman and Ratnieks, 2000). The question whether the distance function continues linearly or progressively flattens over the range of long foraging distances beyond around $1 \mathrm{~km}$, however, is crucial for our biological interpretation of the function (see above). Furthermore, the distance-curve we inferred from our experiment is a representation of how distance is communicated on the level of the colony because we analyzed dances that were each performed by a different bee. We do not know whether the distance-curves of individual bees look the same. An alternative explanation, namely that the non-linear shape of the function on the colony level arose because bees that preferred to forage at closer distances had steeper distance-curves than bees that preferred to forage at longer distances, needs to be excluded. This is crucial given that any inferences about how the bees process distance can only be made based on the distance communication of individual bees. While it was demonstrated in several early studies that the bees can indeed be trained to much further distances (table 1), having the same bees dancing for an array of feeders over a longer range of distances is challenging. In fact, moving a sugar feeder further usually results in a high turnover of foragers. For example, of the 56 bees which we recorded dancing in our experiment, 42 only danced for one feeder $(75 \%), 10$ danced for two feeders $(18 \%)$ and only four danced for three feeders ( 7 $\%)$. To ensure that the same group of bees dances for feeders at several distances, the feeder needs to be moved slowly, ensuring that all bees can keep up. On the other hand, training a colony of honey bees to very far distances, requires quickly moving the feeder forward (von Frisch, 1965). Some experimental tuning needs to be done in future studies to overcome this trade-off.

\section{Implications for waggle dance decoding}

Irrespective of the contribution to basic research, our results have direct implications for an ecological application of the waggle dance. Eavesdropping on waggle dances and decoding their spatial information of is a unique tool to study where honey bee colonies collect forage, and thus to explore how key generalist flower visitors evaluate the landscape (Visscher \& Seeley, 1982; Couvillon, Schürch \& Ratnieks, 2014; Wario et al., 2017; Young et al., 2021). To infer the distances of unknown food sites, researchers have measured either circuit duration (e.g. Visscher and Seeley, 1982; Beekman and Ratnieks, 2000; Steffan-Dewenter and Kuhn, 2003; Danner et al., 2016; Park and Nieh, 2017) or waggle duration (e.g. Couvillon et al., 2015; Bänsch et al., 2020; Carr-Markell et al., 2020) from dances. The use of circuit duration has the advantage that it is faster since it requires only a single time measurement of a sequence of consecutive circuits per dance to obtain a dance's mean circuit duration, and that it can be determined by direct 
398

399

400

401

402

403

404

405

406

407

408

409

410

411

412

413

414

415

416

417

418

419

420

421

422

423

424

425

426

427

428

429

430

431

432

433

434

435

436

437

observation and timing in the field. Furthermore, since Karl von Frisch and his colleagues established an extensive data basis of how circuit duration increases with foraging distance (at least for temperately adapted Carnolian honey bees, A. m. carnica, Fig, 4B (von Frisch, 1965), one can accurately infer the foraging distances of these bees over a long range of potential distances. However, the duration of whole dance circuits includes the duration of the return phase of the dance which is quite variable and conveys little information with respect to foraging distance (Fig 3B). Furthermore, the return phase duration introduces additional variation since it is adjusted by the bees according to resource quality (Seeley, Mikheyev and Pagano, 2000; Hrncir et al., 2011; Łopuch and Tofilski, 2020). Hence, to enhance the precision of foraging distance estimation, it became standard procedure to measure only the actual distance signal of the bees, waggle duration, from dances (Couvillon et al., 2012). Awkwardly, studies that used waggle duration to infer foraging distance relied on linear calibration functions that were experimentally established only over a relatively short range of feeder distances and which did not capture the curvature of the distance-duration relationship (as outlined above). The consequence is that the foraging distances indicated by dances with waggle durations longer than about 1.5-2 seconds (indicating sites further than about 1-1.5 km distance from the hive) were probably systematically underestimated. For example, Frisch and Jander (v. Frisch and Jander, 1957) determined that the mean waggle duration of dances of $A$. mellifera carnica was around four seconds when foraging at a feeder in $4.5 \mathrm{~km}$ distance, but a waggle duration of four seconds translates into a foraging distance of only $2.8 \mathrm{~km}$ when using a recently published distancewaggle duration calibration (Schürch et al., 2019). This inaccuracy is crucial given that typically more than $50 \%$ of foraging in temperate $A$. mellifera takes place beyond $1 \mathrm{~km}$ distance from the hive (Visscher and Seeley, 1982; Beekman and Ratnieks, 2000; Steffan-Dewenter and Kuhn, 2003; Couvillon et al., 2015). We recommend that researchers who plan to do forage mapping should calibrate the distance-waggle duration relationship of their bees by training them to an array of feeder up to distances of at least 1.5 or $2 \mathrm{~km}$, so that the relationship beyond the breakpoint at around $1 \mathrm{~km}$ can be unambiguously established. We also recommend generating a calibration specific to the bees of interest because different populations and species of honey bees can differ in their distance communication functions (Bosch, 1957; Kohl et al., 2020). Such a training experiment (performed during times of little natural nectar flow) will take only about a week time, which is a small fraction of the whole duration needed to complete a forage mapping study. Further, more long-range feeder training studies will lead to a better general understanding of the bees' distance communication function.

\section{Conclusions}

Based on a number of early distance-training experiments, Karl von Frisch and his colleagues concluded that the relationship between foraging distance and waggle duration in the waggle dance of the Western honey bee is well described by a curve with a decreasing positive slope. However, later studies repeatedly found that waggle duration increases linearly with distance, $i$. e. as a straight line, not as a curve. We suspect the results of these later studies are only 
438

439

440

441

442

443

444

445

446

447

448

449

450

451

452

453

454

455

456

457

458

459

460

461

462

463

464

465

466

467

468

469

470

471

472

473

474

475

476

477

478

479

480

481

applicable to distances less than about $1 \mathrm{~km}$, since they are based on experiments in which bees were trained to feeders at relatively short distances. Training bees to longer distances revealed that the distance-related increase in waggle duration is better described by a curve or by a segmented model with two linear segments than by a simple linear regression. Our results can directly be applied to improve the inference of foraging distances based on waggle dance observations. Why the bees' distance communication function has a non-linear shape is a remaining question.

\section{Acknowledgements}

We thank I. Steffan-Dewenter and S. Schiele for their support and for providing the honey bee colony and the equipment used for the experiment. We are grateful to two anonymous reviewers whose valuable comments helped to improve this paper.

\section{References}

Bänsch S, Tscharntke T, Ratnieks FLW, Härtel S, Westphal C. 2020. Foraging of honey bees in agricultural landscapes with changing patterns of flower resources. Agriculture, Ecosystems and Environment 291:106792. DOI: 10.1016/j.agee.2019.106792.

Beekman M, Ratnieks FLW. 2000. Long-range foraging by the honey-bee, Apis mellifera L. Functional Ecology 14:490-496. DOI: 10.1046/j.1365-2435.2000.00443.x.

Bolker B and R Core Team. 2017. bbmle: Tools for General Maximum Likelihood Estimation. R package version 1.0.20. Available at https://cran.r-project.org/package=bbmle

Bosch R. 1957. Rassenmäßige Unterschiede bei den Tänzen der Honigbiene (Apis mellifica L.). Zeitschrift für vergleichende Physiologie 49:289-320.

Carr-Markell MK, Demler CM, Couvillon MJ, Schürch R, Spivak M. 2020. Do honey bee (Apis mellifera) foragers recruit their nestmates to native forbs in reconstructed prairie habitats? PLoS ONE 15:1-20. DOI: 10.1371/journal.pone.0228169.

Clifford CWG, Ibbotson MR. 2002. Fundamental mechanisms of visual motion detection: Models, cells and functions. Progress in Neurobiology 68:409-437. DOI: 10.1016/S03010082(02)00154-5.

Couvillon MJ, Riddell Pearce FC, Accleton C, Fensome K a., Quah SKL, Taylor EL, Ratnieks FLW. 2015. Honey bee foraging distance depends on month and forage type. Apidologie 46:61-70. DOI: 10.1007/s13592-014-0302-5.

Couvillon MJ, Riddell Pearce FC, Harris-Jones EL, Kuepfer AM, Mackenzie-Smith SJ, Rozario LA, Schürch R, Ratnieks FLW. 2012. Intra-dance variation among waggle runs and the design of efficient protocols for honey bee dance decoding. Biology open 1:467-72. DOI: 10.1242/bio.20121099.

Couvillon MJ, Schürch R, Ratnieks FLW. 2014. Dancing Bees Communicate a Foraging Preference for Rural Lands in High-Level Agri-Environment Schemes. Current Biology 24:1212-1215. DOI: 10.1016/j.cub.2014.03.072.

Danner N, Molitor AM, Schiele S, Härtel S, Steffan-Dewenter I. 2016. Season and landscape composition affect pollen foraging distances and habitat use of honey bees. Ecological Applications 26:1920-1929.

Dyer FC. 1991. Comparative studies of dance communication: analysis of phylogeny and function. In: Smith DR ed. Diversity in the Genus Apis. Boulder, CO: Westview, 177-198. 
482 Dyer FC. 2002. The biology of the dance language. Annu. Rev. Entomol. 47:917-49.

483 Dyer FC, Seeley TD. 1991. Dance dialects and foraging range in three Asian honey bee species.

484

485

486

487

488

489

490

491

492

493

494

495

496

497

498

499

500

501

502

503

504

505

506

507

508

509

510

511

512

513

514

515

516

517

518

519

520

521

522

523

524

525

526

527

Behav. Ecol. Sociobiol. 28:227-233.

Esch H, Burns J. 1996. Distance estimation by foraging honeybees. The Journal of experimental biology 199:155-162.

Esch HE, Zhang S, Srinivasan M V, Tautz J. 2001. Honeybee dances communicate distances measured by optic flow. Nature 411:581-3. DOI: 10.1038/35079072.

v. Frisch K, Jander R. 1957. Über den Schwänzeltanz der Bienen. Zeitschrift für Vergleichende Physiologie 40:239-263. DOI: 10.1007/BF00340570.

von Frisch K. 1965. Tanzsprache und Orientierung der Bienen. Berlin, Heidelberg, NewYork: Springer-Verlag.

von Frisch K, Kratky O. 1962. Über die Beziehung zwischen Flugweite und Tanztempo bei der Entfernungsmeldung der Bienen. Die Naturwissenschaften 49:409-417.

George EA, Thulasi N, Kohl PL, Suresh S, Rutschmann B, Brockmann A. 2020. Do honey bee species differ in the odometer used for the waggle dance? bioRxiv:2020.12.17.423277. DOI: 10.1101/2020.12.17.423277.

Heran H. 1956. Ein Beitrag zur Frage nach der Wahrnehmunngsgrundlage der Entfernungsweisung der Bienen (Apis mellifica L.). Zeitschrift für Vergleichende Physiologie 38:168-218.

Hrncir M, Maia-Silva C, Mc Cabe SI, Farina WM. 2011. The recruiter's excitement - features of thoracic vibrations during the honey bee's waggle dance related to food source profitability. Journal of Experimental Biology 214:4055-4064. DOI: 10.1242/jeb.063149.

Kohl PL, Thulasi N, Rutschmann B, George EA, Steffan-Dewenter I, Brockmann A. 2020. Adaptive evolution of honeybee dance dialects. Proceedings of the Royal Society B: Biological Sciences 287. DOI: 10.1098/rspb.2020.0190.

Lindauer M. 1956. Über die Verständigung bei indischen Bienen. Zeitschrift für vergleichende Physiologie 38:521-557

Łopuch S, Tofilski A. 2020. Impact of the quality of food sources on the wing beating of honey bee dancers. Apidologie 51:631-641. DOI: 10.1007/s13592-020-00748-3.

Maddess T, Laughlin SB. 1985. Adaptation of the motion-sensitive neuron H1 is generated locally and governed by contrast frequency. Proceedings of the Royal Society of London Biological Sciences 225:251-275. DOI: 10.1098/rspb.1985.0061.

De Marco R, Menzel R. 2005. Encoding spatial information in the waggle dance. Journal of Experimental Biology 208:3885-3894. DOI: 10.1242/jeb.01832.

Michelsen A, Andersen BB, Storm J, Kirchner WH, Lindaner M. 1992. How honeybees perceive communication dances, studied by means of a mechanical model. Behav. Ecol. Sociobiol. 30:143-150.

Park B, Nieh JC. 2017. Seasonal trends in honey bee pollen foraging revealed through DNA barcoding of bee-collected pollen. Insectes Sociaux 64:425-437. DOI: 10.1007/s00040-0170565-8.

Preece K, Beekman M. 2014. Honeybee waggle dance error: adaption or constraint? Unravelling the complex dance language of honeybees. Animal Behaviour 94:19-26. DOI: 10.1016/j.anbehav.2014.05.016.

R Core Team. 2019. R: A Language and Environment for Statistical Computing. Vienna, Austria: R Foundation for Statistical Computing.

Sasaki M, Takahashi H, Sato T. 1993. Comparison of the dance dialect and foraging range

PeerJ reviewing PDF | (2020:12:56277:1:1:NEW 7 Mar 2021) 
528

529

530

531

532

533

534

535

536

537

538

539

540

541

542

543

544

545

546

547

548

549

550

551

552

553

554

555

556

557

558

559

560

561

562

563

564

565

566

567

568

569

570

571

572

573 between Apis mellifera and northenmost subspecies of A. cerana in Japan. Honeybee Science 14:49-52.

Schneider SS. 1989. Spatial foraging patterns of the African honey bee, Apis mellifera scutellata. Journal of Insect Behavior 2:505-521. DOI: 10.1007/BF01053351.

Schürch R, Couvillon MJ, Burns DDR, Tasman K, Waxman D, Ratnieks FLW. 2013. Incorporating variability in honey bee waggle dance decoding improves the mapping of communicated resource locations. Journal of Comparative Physiology A 199:1143-1152. DOI: $10.1007 / \mathrm{s} 00359-013-0860-4$.

Schürch R, Ratnieks FLW, Samuelson EEW, Couvillon MJ. 2016. Dancing to her own beat: honey bee foragers communicate via individually calibrated waggle dances. The Journal of experimental biology 219:1287-1289. DOI: 10.1242/jeb.134874.

Schürch R, Zwirner K, Yambrick BJ, Pirault T, Wilson JM, Couvillon MJ. 2019. Dismantling Babel: creation of a universal calibration for honey bee waggle dance decoding. Animal Behaviour 150:139-145.

Seeley TD, Mikheyev AS, Pagano GJ. 2000. Dancing bees tune both duration and rate of waggle-run production in relation to nectar-source profitability. Journal of Comparative Physiology - A Sensory, Neural, and Behavioral Physiology 186:813-819. DOI: $10.1007 / \mathrm{s} 003590000134$.

Si A, Srinivasan M V., Zhang S. 2003. Honeybee navigation: Properties of the visually driven “odometer." Journal of Experimental Biology 206:1265-1273. DOI: 10.1242/jeb.00236.

Srinivasan M V. 2000. Honeybee Navigation: Nature and Calibration of the "Odometer." Science 287:851-853. DOI: 10.1126/science.287.5454.851.

Steche W. 1957. Zur Analyse Der Bienentänze (Teil I). Insectes Sociaux 4:305-318.

Steffan-Dewenter I, Kuhn A. 2003. Honeybee foraging in differentially structured landscapes. Proceedings. Biological sciences / The Royal Society 270:569-75. DOI: 10.1098/rspb.2002.2292.

Su S, Cai F, Si A, Zhang S, Tautz J, Chen S. 2008. East learns from West: Asiatic honeybees can understand dance language of European honeybees. PloS one 3:e2365. DOI: 10.1371/journal.pone.0002365.

Tautz J, Zhang S, Spaethe J, Brockmann A, Si A, Srinivasan M. 2004. Honeybee Odometry: Performance in Varying Natural Terrain. PLoS Biology 2:e211. DOI: 10.1371/journal.pbio.0020211.

Taylor GK, Krapp HG. 2007. Sensory Systems and Flight Stability: What do Insects Measure and Why? DOI: 10.1016/S0065-2806(07)34005-8.

Visscher PK. 1982. Foraging strategy of honey bee colonies in a temperate deciduous forest. Cornell University, Ithaka, New York, USA.

Visscher PK, Seeley TD. 1982. Foraging Strategy of Honeybee Colonies in a Temperate Deciduous Forest. Ecology 63:1790-1801.

Vito M, Muggeo R. 2008. segmented: an R Package to Fit Regression Models with Broken-Line Relationships. R News 8:20-25.

Waddington KD, Visscher PK, Herbert TJ, Raveret M. 1994. Comparisons of forager distributions from matched in suburban honey bee colonies environments. Behavioral Ecology and Sociobiology 35:423-429. DOI: 10.1007/BF00165845.

Wario F, Wild B, Rojas R, Landgraf T. 2017. Automatic detection and decoding of honey bee waggle dances. PLoS ONE 12:1-16. DOI: 10.1371/journal.pone.0188626.

Wenner AM. 1962. Sound production during the waggle dance of the honey bee. Animal 
574 Behaviour 10:79-95. DOI: 10.1016/0003-3472(62)90135-5.

575 Young AM, Kohl PL, Rutschmann B, Steffan-Dewenter I, Brockmann A, Dyer FC. 2021.

576 Temporal and spatial foraging patterns of three Asian honey bee species in Bangalore,

577 India. Apidologie. https://doi.org/10.1007/s13592-020-00839-1 


\section{Figure 1}

Potential curvature in the distance communication function

(A) Dance circuit duration in A. mellifera carnica as a function of feeder distance. Data are average values presented by von Frisch (1965) in his synopsis on the dance language (original data are up to $9.5 \mathrm{~km}$ ). The distance-circuit duration relationship deviates from a simple linear shape (the line is a segmented regression with a break-point at $0.7 \mathrm{~km}$ distance). (B) Analysis of published feeder training experiments with respect to whether the distance communication function was found to be non-linear (y-axis) as a function of how far the bees were trained away from the hive (x-axis). Colours indicate whether researchers timed the waggle duration (the actual distance signal, red) or the circuit duration (waggle phase plus return phase, a proxy of the distance signal, lightblue). The dashed line is a logistic regression model. The studies considered are listed in table 1 (study number eleven is not considered in this figure, and the study by Lindauer, 1960, who trained bees up to 11 $\mathrm{km}$ from the hive is outside of the plotting range of the figure).
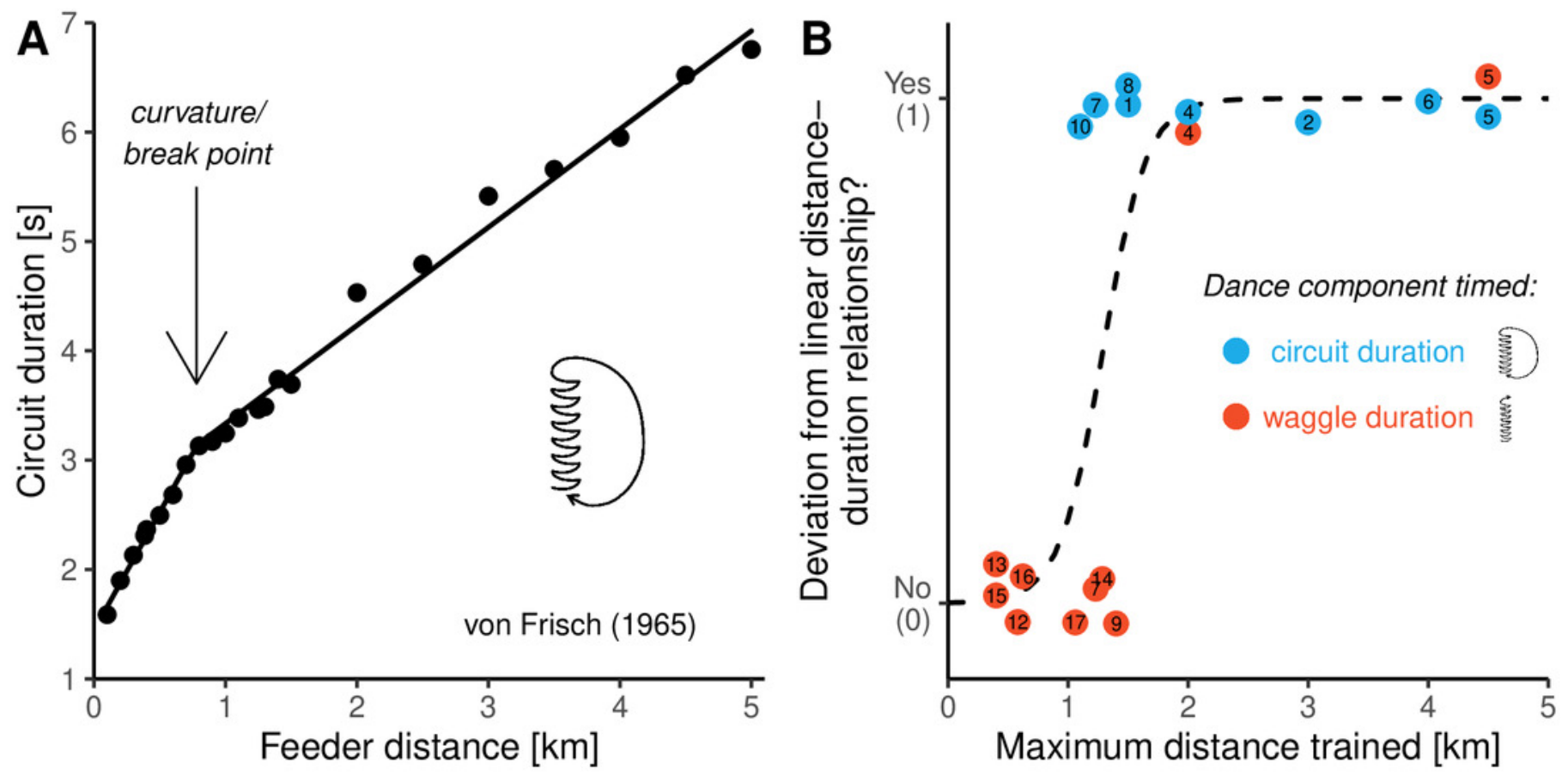


\section{Figure 2}

Results of two historical studies exploring the relationships between feeder distance and the duration of three components of the waggle dance in A. mellifera

Red: waggle phase, dark blue: return phase, light blue: whole dance circuit. The main graph shows the curves obtained by von Frisch and Jander (1957) who trained bees up to $4.5 \mathrm{~km}$ from the hive. The small graph embedded in the top left corner shows the curves obtained by Wenner (1962) who trained bees up to $1.23 \mathrm{~km}$ from the hive. The graphs are scaled equally. In both studies, authors report that the circuit duration function is non-linear, however, while von Frisch and Jander's data suggest that the curvature stems from the waggle phase, Wenner's data suggest that it stems from the return phase of the dance. 


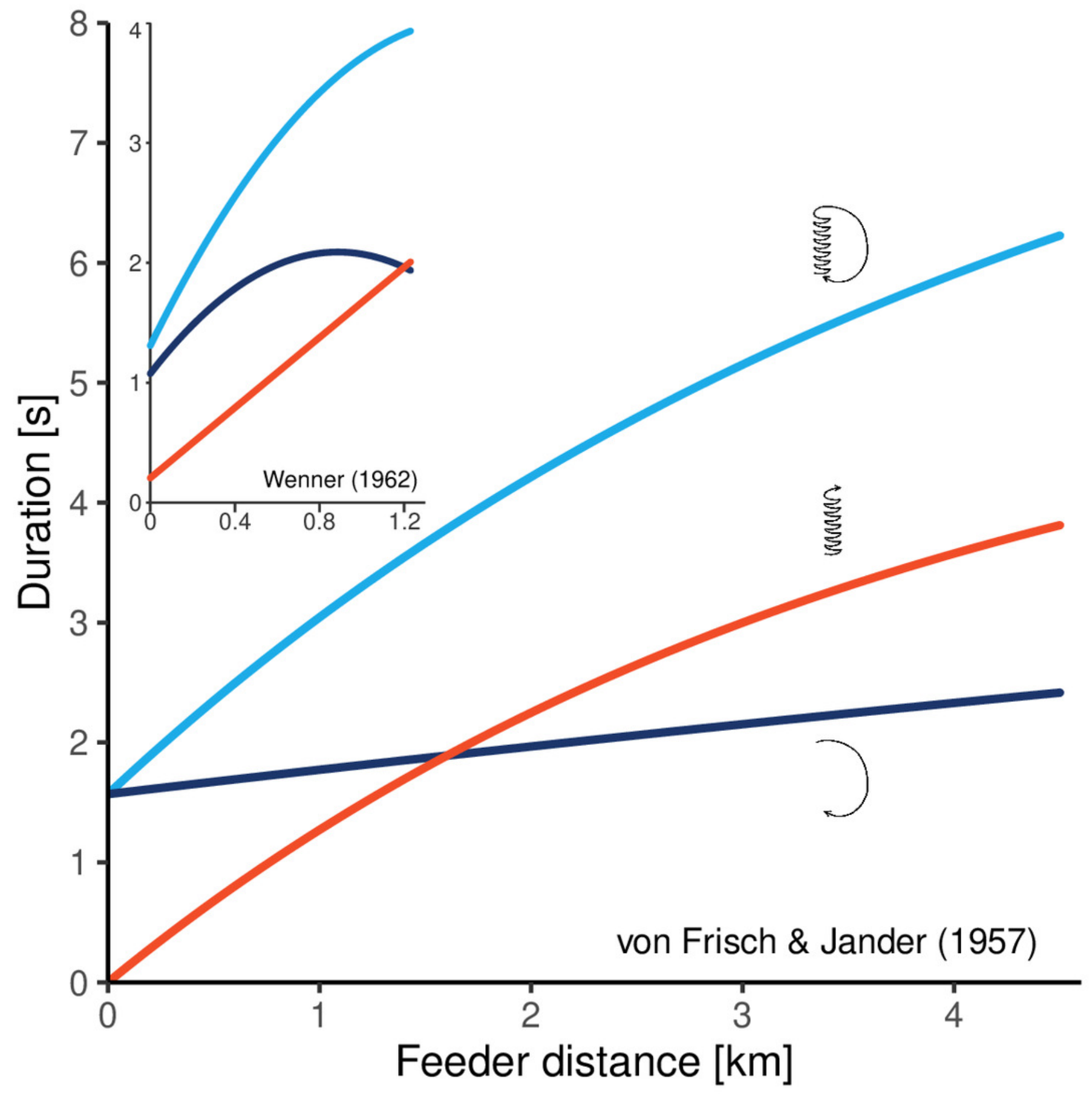


Figure 3

(A) Waggle phase duration, (B) return phase duration and (C) circuit duration of waggle dances performed by bees trained to feeders at distances between 0.1 and $1.7 \mathrm{~km}$ in this study.

Open circles are raw data (mean values per dance, each dance by a different bee), solid black dots are mean values per distance, and lines represent the predictions of the best models (based on AICC) for each component (see table 2).
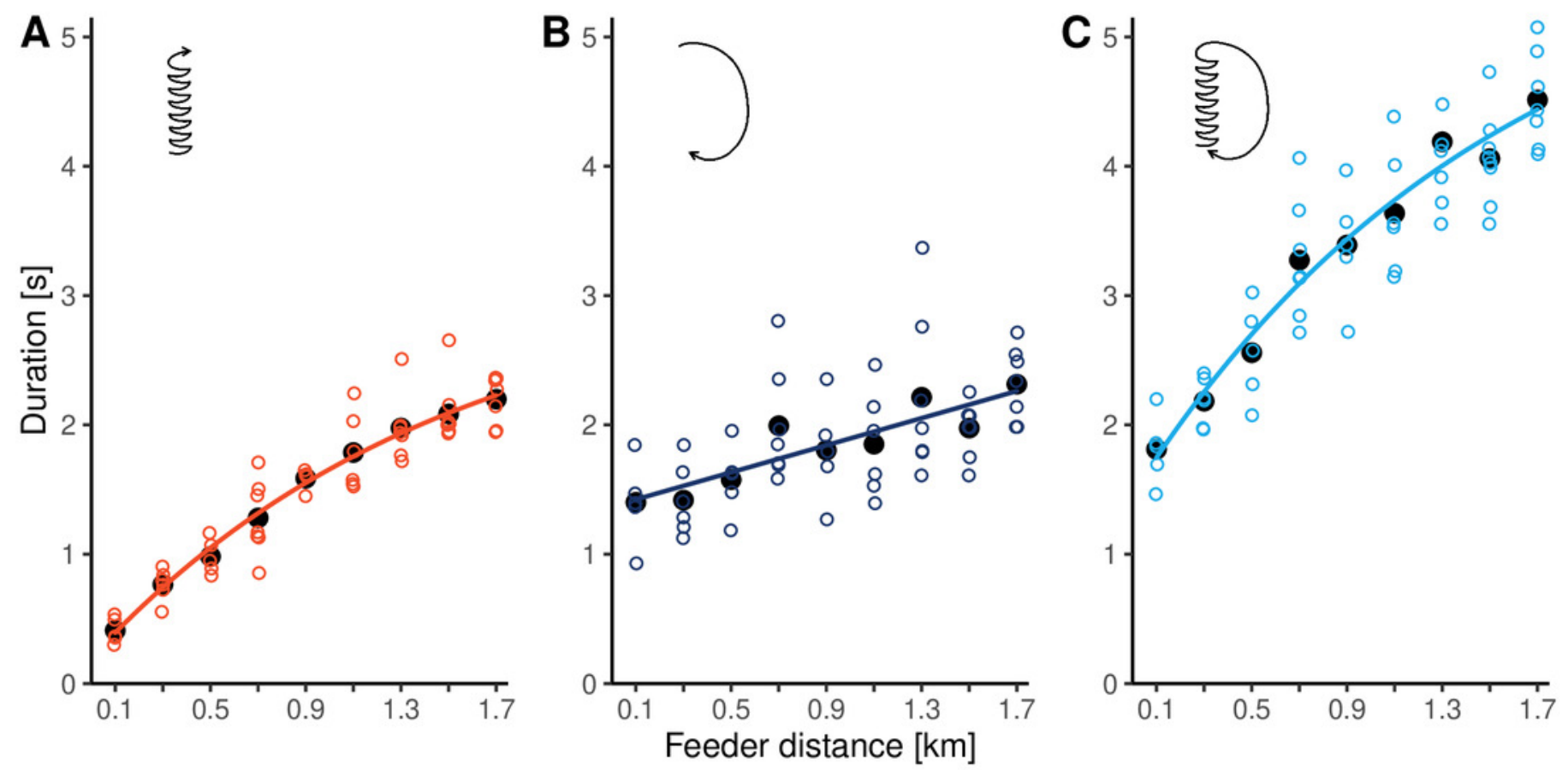


\section{Figure 4}

\section{Comparisons of distance-duration curves}

(A) The relationship between waggle duration and feeder distance obtained in this study (red dots, mean values per distance) compared to the results of von Frisch and Jander (1957) (black dots, mean values per distance). Data from this study are modelled by the non-linear function for the data range up to $1.7 \mathrm{~km}$ (solid red line, the same model as presented in Fig. $3 \mathrm{~A}$, see table 2) and extrapolated with the second segment of the segmented model (dashed red line, see table 2). The data of Frisch and Jander were remodeled by us with a non-linear model up to $1.4 \mathrm{~km}$ distance and with a linear model beyond (solid black line; for $d \leq 1.4 \mathrm{~km}$ : $t_{w}=0.1096+1.7208 / 0.6272 *\left(1-\mathrm{e}^{-0.6272^{* d}}\right)$, for $\left.d>1.4 \mathrm{km:} t_{w}=0.7198069+0.70947 * d\right)$. The yellow line represents the linear model presented by Schürch et al (2019) which is based on several feeder training experiments (the solid line segment represent the range of distances up to $1.285 \mathrm{~km}$, for which data were available; the dashed line segment is the extrapolation). (B) The relationship between circuit duration and feeder distance obtained in this study (light blue dots, mean values per distance) compared to data presented by von Frisch (1965) (black dots, average data from several studies per distance). The solid light blue line up to $1.7 \mathrm{~km}$ stems from the non-linear model based on our data (the same model as presented in Fig. 3C, see table 2). The dashed line is a linear extrapolation based on the values presented by von Frisch (formula: $t_{c}=3.072032+0.8046 * d$ ). 

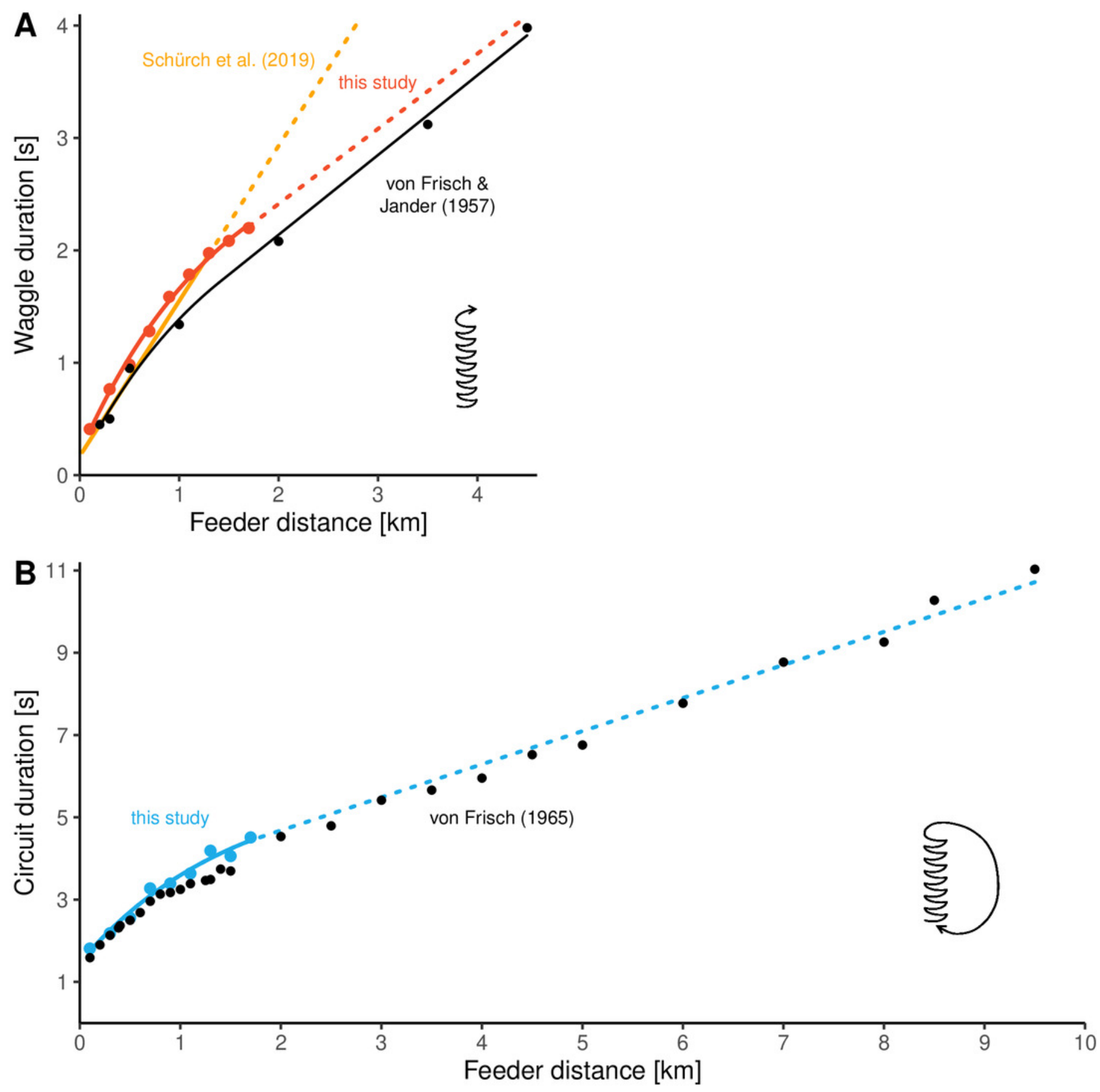


\section{Table $\mathbf{1}$ (on next page)}

Published distance-training experiments with temperately adapted European $A$. mellifera.

The study IDs are the same as used for the numbering of data points in figure 1A. Information is provided on the bees used, the location of the experiment, the maximum distance to which the bees were trained, the components of the waggle dance measured, and whether the observed relationship between feeder distance and the duration of the dance component(s) deviated from linearity. The last column contains the references. 
Table 1. Published distance-training experiments with temperately adapted European A. mellifera. The study IDs are the same as used for the numbering of data points in figure 1A. Information is provided on the bees used, the location of the experiment, the maximum 3 distance to which the bees were trained, the components of the waggle dance measured, and whether the observed relationship 4 between feeder distance and the duration of the dance component(s) deviated from linearity. The last column contains the references.

\begin{tabular}{|c|c|c|c|c|c|c|}
\hline $\begin{array}{l}\text { Study } \\
\text { ID }\end{array}$ & $\begin{array}{l}\text { Honeybee } \\
\text { subspecies }\end{array}$ & Location & $\begin{array}{c}\text { Max. } \\
\text { distance } \\
\text { trained }(\mathbf{k m})\end{array}$ & $\begin{array}{c}\text { Dance } \\
\text { component(s) } \\
\text { timed }\end{array}$ & $\begin{array}{l}\text { Deviation from } \\
\text { linear distance- } \\
\text { duration } \\
\text { relationship? }\end{array}$ & Reference \\
\hline 1 & A. m. carnica & Brunnwinkl, Austria & 1.5 & dance circuit & yes & v. Frisch 1945 (in v. Frisch 1965, p. 66) \\
\hline 2 & A. m. carnica & St. Gilgen, Austria & 3 & dance circuit & yes & v. Frisch 1946 (in v. Frisch 1965, p. 66) \\
\hline 3 & A. m. carnica & Graz, Austria & 11 & dance circuit & yes & $\begin{array}{l}\text { Knaffl \& Lindauer } 1949 \text { (in v. Frisch 1965, p. } \\
67 \text { ) }\end{array}$ \\
\hline 4 & A. m. carnica & Bonn, Germany & 2 & $\begin{array}{l}\text { waggle phase } \\
\text { dance circuit }\end{array}$ & $\begin{array}{l}\text { yes } \\
\text { yes }\end{array}$ & Steche 1957 \\
\hline 5 & A. m. carnica & Munich, Germany & 4.5 & $\begin{array}{l}\text { waggle phase } \\
\text { dance circuit }\end{array}$ & $\begin{array}{l}\text { yes } \\
\text { yes }\end{array}$ & v. Frisch \& Jander 1957 \\
\hline 6 & A. m. carnica & Munich, Germany & 4 & dance circuit & yes & Lindauer 1960 (in v. Frisch 1965, p. 66) \\
\hline 7 & A. m. ligustica & Pinckney, Michigan, USA & 1.23 & $\begin{array}{l}\text { waggle phase } \\
\text { dance circuit }\end{array}$ & $\begin{array}{l}\text { no } \\
\text { yes }\end{array}$ & Wenner 1962 \\
\hline 8 & A. m. sspp.-hybrid & $\begin{array}{l}\text { Arnot Forest, New York, } \\
\text { USA }\end{array}$ & 1.5 & dance circuit & yes & Visscher 1982 \\
\hline 9 & A. m. ligustica-hybrid & Japan & 1.4 & waggle phase & no & Sasaki et al. 1993 \\
\hline 10 & A.m.sspp.-hybrid & Florida, USA & 1.1 & dance circuit & yes & Waddington et al. 1994 \\
\hline 11 & A. m. carnica & Göttingen, Germany & 1 & dance circuit & yes* & Steffan-Dewenter \& Kuhn 2003 \\
\hline 12 & A. m. sspp.-hybrid & Canberra, Australia & 0.58 & waggle phase & no & Tautz et al. 2004 \\
\hline 13 & A. m. ligustica & Hangzhou, China & 0.4 & waggle phase & no & Su et al. 2008 \\
\hline 14 & A. m. mellifera-hybrid & Brighton, UK & 1.285 & waggle phase & no & Schürch et al. 2013 \\
\hline 15 & A. m. mellifera-hybrid & Brighton, UK & 0.4 & waggle phase & no & Schürch et al. 2016 \\
\hline 16 & A. m. ligustica-hybrid & Blacksburg, Virginia, USA & 0.622 & waggle phase & no & Schürch et al. 2019 \\
\hline 17 & A. m. ligustica-hybrid & Afton, Minnesota, USA & 1.06 & waggle phase & no & Carr-Markell et al. 2020 \\
\hline
\end{tabular}


5 * The results of Steffan-Dewenter and Kuhn (2003) suggest a non-linear relationship of distance and circuit duration, but since bees were tested at a low number of feeder 6 distances (ca. 260, 500 and $1000 \mathrm{~m}$ from the hive), the shape of the distance-duration function cannot be unambiguously determined. 


\section{Table 2 (on next page)}

Model comparisons for the distance-duration relationships

Comparison of non-linear, segmented linear and simple linear models of the relationships between feeder distance $(d$, in $\mathrm{km})$ and the duration of the waggle phase $\left(t_{w}\right)$, the return phase $\left(t_{r}\right)$ and the whole dance circuit $\left(t_{c}\right)$ (each in $s$ ) based on the data obtained in this study. The models are ordered according to their $\triangle A I C c$ values (the "best" models are highlighted in bold). 
1 Table 2. Comparison of non-linear, segmented linear and simple linear models of the 2 relationships between feeder distance $(d$, in $\mathrm{km})$ and the duration of the waggle phase $\left(t_{w}\right)$, the 3 return phase $\left(t_{r}\right)$ and the whole dance circuit $\left(t_{c}\right)$ (each in s) based on the data obtained in this 4 study. The models are ordered according to their $\triangle \mathrm{AICc}$ values (the "best" models are 5 highlighted in bold).

\begin{tabular}{|c|c|c|c|c|c|}
\hline Dance component & Model & Is is biased?§ & Formula & $r^{\#}$ & $\Delta \mathrm{AICc}$ \\
\hline \multirow{3}{*}{ Waggle phase } & non-linear & no & $t_{w}=0.1993+\frac{2.0018}{0.6717}\left(1-e^{-0.6717 * d}\right)$ & 0.946 & $\mathbf{0}$ \\
\hline & segmented & no & $t_{w}=\left\{\begin{array}{r}0.2917+1.4282 * d, d \leq 1.0328 \\
1.0767+0.6683 * d, d>1.0328\end{array}\right.$ & 0.947 & 1.5 \\
\hline & linear & yes & $t_{w}=0.4475+1.1152 * d$ & 0.935 & 7.7 \\
\hline \multirow{3}{*}{ Return phase } & linear & no & $t_{r}=1.3712+0.5238 * d$ & 0.582 & $\mathbf{0}$ \\
\hline & non-linear & no & $t_{r}=1.2686+\frac{0.8876}{0.6035}\left(1-e^{-0.6035 * d}\right)$ & 0.587 & 1.9 \\
\hline & segmented & no & $t_{r}=\left\{\begin{array}{l}1.2303+0.8613 * d, d \leq 0.7 \\
1.5725+0.3725 * d, d>0.7\end{array}\right.$ & 0.596 & 3.4 \\
\hline \multirow{3}{*}{ Dance circuit } & non-linear & no & $t_{c}=1.467+\frac{2.893}{0.6519}\left(1-e^{-0.6519 * d}\right)$ & 0.904 & $\mathbf{0}$ \\
\hline & segmented & no & $t_{c}=\left\{\begin{array}{l}1.4926+2.4201 * d, d \leq 0.7 \\
2.2844+1.2889 * d, d>0.7\end{array}\right.$ & 0.906 & 1.4 \\
\hline & linear & yes & $t_{c}=1.8187+1.6390 * d$ & 0.894 & 2.6 \\
\hline
\end{tabular}

6 § Does the model provide biased predictions, i. e. do the predictions systematically deviate from the actual observations at any

7 point over the range of feeder distances? (Decision aided by the inspection of plots of fitted values versus residuals)

8 \# Pearson's $r$ of a correlation between fitted and observed values, a measure of how well the models fit the data 